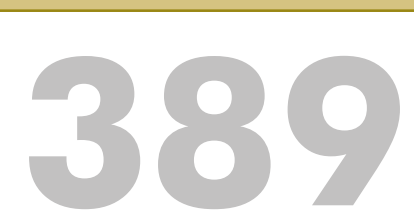

Março 2010

COPULA BASED MODELS

FOR

SERIAL DEPENDENCE

Beatriz Vaz de Melo Mendes Ceclia Aíube 
Relatórios COPPEAD é uma publicação do Instituto COPPEAD de Administração da Universidade Federal do Rio de Janeiro (UFRJ)

\section{Editor}

Prof. Mauricio Mittelman

\section{Assistente do Editor}

Lucilia Silva

\section{Ficha Catalográfica}

Ana Rita Mendonça de Moura

Mendes, Beatriz Vaz de Melo.

Copula based models for serial dependence / Beatriz Vaz de Melo Mendes e Cecilia Aíube. - Rio de Janeiro: UFRJ/COPPEAD, 2010.

18 p.; $27 \mathrm{~cm}$. - (Relatórios Coppead; 389)

ISBN 978-85-7508-076-4

ISSN 1518-3335

1. Finanças. I. Título. II. Série.

CDD - 332

\section{Pedidos para Biblioteca}

Caixa Postal 68514 - Ilha do Fundão

21941-972 - Rio de Janeiro - RJ

Telefone: $21-2598-9837$

Telefax: 21-2598-9835

e-mail: biblioteca@coppead.ufri.br

Site: www.coppead.ufri.br 


\title{
Copula Based Models for Serial Dependence
}

\author{
Beatriz Vaz de Melo Mendes ${ }^{1}$ \\ IM/COPPEAD, Federal University at Rio de Janeiro, Brazil.
}

Cecília Aíube

COPPEAD, Federal University at Rio de Janeiro, Brazil.

JEL subject classifications: C51 C22 G19

Keywords: Copulas; Time series conditional models; Tail dependence; Value-atRisk.

\begin{abstract}
This paper is concerned with the statistical modeling of the dependence structure in the first and second moments of a univariate financial time series using the concept of copulas. The appealing feature of the method is that it captures not just the linear form of dependence (a job usually accomplished by ARIMA linear models), but also the non-linear ones, including tail dependence, the dependence occuring only among extreme values. In addition we investigate the changes in the mean modeling after whitening the data through the application of GARCH type filters. Sixty two U.S. stocks are selected to illustrate the methodologies. The copula based results corroborate empirical evidences on the existence of linear and non-linear dependence at the mean and at the volatility levels, and contributes to practice by providing yet a simple but powerful method for capturing the dynamics in a time series. Applications may follow and include VaR calculation, simulations based derivatives pricing, and asset allocation decisions. We recall that the literature is still inconclusive as to the most appropriate Value-at-Risk computing approach, which seems to be a data dependent decision.
\end{abstract}

\footnotetext{
${ }^{1}$ Corresponding author. beatriz im.ufrj.br.
} 


\section{Introduction}

This paper is concerned with the statistical modeling of the temporal dependence structure existing in a univariate financial series using the concept of copulas.

Modeling the dependence structure among variables using copulas is an approach recently rediscovered (see Joe (1997) and Nelsen (2006), Genest, Gendron and Bourdeau-Brien (2008) for a review on the subject). For every continuous multivariate distribution there is a unique copula which links the marginal variables, isolating the description of their dependence structure independently of the marginal distributions. Modeling multivariate data through copulas adds flexibility, since there is a uncountable number of possible combinations of copulas families and marginal distributions that can be assumed.

Copulas had become popular in finance mostly in the bivariate context (see Cherubini, Luciano, and Vecchiato (2004), Mc Neil, Frey, and Embretchs (2005), and references therein). However, copulas can also be used to model the temporal dependence structures in a univariate time series $r_{1}, r_{2}, \cdots, r_{T}$. Instead of a pair of assets, we now consider the bivariate series formed by pairs of consecutive returns $\left(r_{1}, r_{2}\right),\left(r_{2}, r_{3}\right), \cdots,\left(r_{T-1}, r_{T}\right)$, an idea first suggested in Joe (1997) and pursued later by Domma, Giordano and Perri (2001), Chen and Fan (2006), and Ibragimov (2009). Since an appropriate copula function can be found for any type of association (linear, non-linear, ranging from perfect negative to perfect positive dependence), modeling the serial dependence within a univariate series using copulas brings new contributions to the time series theory. We recall that the well known ARIMA processes, designed to model a time series conditional mean, are built up solely based on the behavior of the linear correlation between lagged observations.

In this paper we model the temporal dynamics in the first and second moments of a financial returns series using the concept of copulas. Initially, we treat the returns series as a first order Markov process, and model the conditional mean by fitting copulas to the bivariate series of consecutive returns. Differently from Domma, Giordano e Perri (2001), here we use the skew- $t$ distribution for modeling the unconditional distribution of the returns and elliptical copulas. The skew- $t$ allows for 
high kurtosis and asymmetry, providing tailored marginal fits, improvements carried on to the conditional copula fit. The copula families used in this paper are able to model linear and non-linear dependence, as well as temporal association occuring only between extreme values, and as the copula parameter ranges in its parameter space, all dependence measures change accordingly.

Next, we treat the squared returns as a first order Markov process, and model the dynamics in the conditional volatility by fitting copulas to pairs of consecutive squared returns $\left(r_{1}^{2}, r_{2}^{2}\right),\left(r_{2}^{2}, r_{3}^{2}\right), \cdots,\left(r_{T-1}^{2}, r_{T}^{2}\right)$. This approach can be seen as an alternative to the $\mathrm{ARCH}(1)$ model. However, differently from the $\mathrm{ARCH}(1)$ process, under which the squared returns follow an autoregressive process of order 1, under the conditional copula approach the whole dependence structure linking consecutive squared returns is specified, and not just the lag 1 (linear) autocorrelation.

Finally, we investigate whether or not the dynamic behavior of the series' second moment spills over the behavior of the first moment. In other words, we investigate if any type of serial dependence in the mean is left over after filtering the data through some GARCH type model, verifying if the dependence in the level of consecutive residuals (filtered returns) is in anyway different from that initially found for the (raw) returns.

In Section 2 we present a brief review of the copula theory and provide the theoretical background for the univariate conditional modeling using copulas. In Section 3 we provide an real data example based on daily returns of 62 stocks from the New York Stock Exchange (NYSE), chosen from 8 different sectors: Conglomorates, Consumer Goods, Financial, Healthcare, Ind. Goods, Services, Technology, Utilities. For each sector we pick the 5 largest and the 5 smallest stocks in terms of market capitalization. We empirically show that the estimates and forecasts of the conditional mean and volatility can be improved when the ascertained dependence between consecutive returns is properly described through a copula function. This is assessed through an out-of-sample Value-at-Risk computation. Results indicate that when returns and/or squared returns possess tail dependence, the conditional copula strategy may result in better out-of-sample VaR estimates performance. Some concluding remarks are given in Section 4. 


\section{Copulas: a brief review}

\section{$2.1 \quad$ Copulas}

Let $F$ represent the joint distribution function $(d f)$ of a continuous random vector $X=\left(X_{1}, \ldots, X_{d}\right) \in R^{d}$. Let $F_{1}, \ldots, F_{d}$ be their marginal $d f \mathrm{~s}$, and let $X_{i} \rightarrow$ $F_{i}\left(X_{i}\right), i=1, \ldots, d$, be their probability integral transformation to the standard uniform distribution. Then the copula $C$ pertaining to $F$ is defined as

$$
C\left(u_{1}, \ldots, u_{d}\right)=F\left(F_{1}^{-1}\left(u_{1}\right), \ldots,\left(F_{d}^{-1}\left(u_{d}\right)\right), \quad \forall\left(u_{1}, \ldots, u_{d}\right) \in(0,1)^{d}\right.
$$

where $F_{i}^{-1}$ is the quantile function of $F_{i}, i=1, \ldots, d$. Thus the copula is a multivariate distribution with uniform $(0,1)$ margins. The copula $C$ completely specifies the distribution $F$ (Sklar, 1996) inasmuch as $\forall\left(x_{1}, \ldots, x_{d}\right) \in R^{d}$

$$
F\left(x_{1}, \ldots, x_{d}\right)=C\left(F_{1}\left(x_{1}\right), \ldots, F_{n}\left(x_{d}\right)\right) .
$$

When $F$ is not continuous, there still exists the copula representation of $F$, but it may not be unique anymore (Schweizer \& Sklar, 1983). The copula of any $F$ captures and summarizes the dependence between the $X_{i}$ variables, and is invariant under strictly increasing transformations of the $X_{i}$.

By taking partial derivatives of (2) one obtains the multivariate density function

$$
f\left(x_{1}, \cdots, x_{d}\right)=c\left(F_{1}\left(x_{1}\right), \cdots, F_{d}\left(x_{d}\right)\right) \prod_{i=1}^{d} f_{i}\left(x_{i}\right)
$$

where $c$ denotes a $d$-dimensional copula density. Decomposition (3) simplifies the specification of the data underlying multivariate distribution and suggests estimating the marginal distributions $f_{i}$ separated from the dependence structure given by the $d$-variate copula.

Dependence measures defined only through $C(\cdot)$ or $c(\cdot)$ whose expressions do not involve the margins $F_{i}$ or $f_{i}$, are known as copula based dependence measures. An important copula based dependence measure is the tail dependence coefficient. The concept of bivariate tail dependence relates to the amount of dependence in the upper- or lower-quadrant tails of a bivariate distribution. The tail dependence 
coefficient is a number in $[0,1]$ and measures dependence between extreme joint gains $\left(\lambda_{U}\right)$ or losses $\left(\lambda_{L}\right)$. The upper and lower tail dependence coefficients are respectively given by

$$
\lambda_{U}=\lim _{u \rightarrow 1} \frac{\bar{C}(u, u)}{1-u}
$$

and

$$
\lambda_{L}=\lim _{u \rightarrow 0} \frac{C(u, u)}{u},
$$

if these limits exist, and where $\bar{C}(u, v)$ is the survival copula, $\bar{C}(u, v)=C(1-u, 1-$ $v)$.

From now on we assume $d=2$. In the bivariate case all copula functions satisfy the following inequality

$$
\max (u+v-1,0) \leq C(u, v) \leq \min (u, v)
$$

where the bounds are copulas of functionally dependent variables. The lower bound, the minimum copula denoted by $C^{-}$, models perfect negative dependence, while the upper bound, the maximum copula denoted by $C^{+}$, models perfect positive dependence. Some copula families cover the entire range of dependence, from $C^{-}$ to the product copula $C^{I}$, finally reaching $C^{+}$, while the indexing parameter ranges over the parameter space, see Table $1 . C^{I}$ is the copula of independent variables and it serves as basis (null hypothesis) for the generalized log-likelihood test.

Archimedean copulas are popular in finance and are particularly easy to handle (see Genest and MacKay, 1986). They are based on a generating function $\phi$ : $[0,1] \rightarrow[0, \infty)$ continuous, strictly decreasing, convex and such that $\phi(1)=0$. Given such a $\phi$ function, a bivariate Archimedean copula may be constructed from $C(u, v)=\phi^{-1}(\phi(u)+\phi(v))$. The class of one parameter Archimedean copulas includes the Gumbel, Frank, Clayton, and Joe copulas. Two-parameter families include the BB1, BB2, BB3, BB6 and BB7 copulas (notation of Joe, 1997). Details on the Archimedean copulas may be found in Joe (1997). The two-parameter BB7 (also known as Joe-Clayton) family allows for modeling different strengths of upper 
and lower tail dependences, an appealing feature since in finance major emphasis is given to the dependence in joint extreme losses.

The elliptical copulas are those pertaining to elliptical families. Most popular members are the Gaussian and the t-copula. Both are parameterized by the linear correlation coefficient $\rho$ and the t-copula has a second parameter, $\nu$, the degrees of freedom. The symmetric t-copula possess $\lambda_{L}=\lambda_{U}$ which is non-zero even when $\rho=0.0$.

A summary of the copulas used in this paper, their family and type, parameters and their limiting special cases, along with the value of their tail dependence coefficients, are given in Table 1. The limit copulas are the copulas attained when the parameter(s) reaches the parameter space boundaries. For all these copulas the strengh of dependence increases with $\rho$ or $\theta$.

Table 1: Summary characteristics of selected copulas. For the t-copula, $\lambda_{L}=\lambda_{U}=$ $2 t_{\nu+1}(-\sqrt{\nu+1} \sqrt{1-\rho} / \sqrt{1+\rho})$. The Gumbel copula has $\lambda_{U}=2-2^{1 / \theta}$. Clayton copula possess $\lambda_{L}=2^{-1 / \theta}$. For the BB7 copula, $\lambda_{U}=2-2^{1 / \theta}$ may be different from $\lambda_{L}=2^{-1 / \delta}$.

\begin{tabular}{lccccccc}
\hline \hline Family & Type & Parameters & $\lambda_{L}$ & $\lambda_{U}$ & $C^{-}$ & $C^{I}$ & $C^{+}$ \\
\hline Gaussian & Elliptical & $-1 \leq \rho \leq+1$ & 0.0 & 0.0 & $\rho=-1$ & $\rho=0$ & $\rho=+1$ \\
\hline t-copula & Elliptical & $-1 \leq \rho \leq+1, \nu$ & $\sqrt{ }$ & $\sqrt{ }$ & $\rho=-1$ & - & $\rho=+1$ \\
\hline Frank & Archim. & $-\infty<\theta<+\infty$ & 0.0 & 0.0 & $\theta \rightarrow-\infty$ & $\theta=0$ & $\theta \rightarrow+\infty$ \\
\hline Surv.Clayton & Archim./EV & $\theta \geq 0$ & 0.0 & $\sqrt{ }$ & - & $\theta=1$ & $\theta \rightarrow+\infty$ \\
\hline Gumbel & Archim./EV & $\theta \geq 1$ & 0.0 & $\sqrt{ }$ & - & $\theta=1$ & $\theta \rightarrow+\infty$ \\
\hline BB7 & Archimedean & $\theta \geq 1, \delta>0$ & $\sqrt{ }$ & $\sqrt{ }$ & - & - & $\theta$ or $\delta \rightarrow \infty$ \\
\hline \hline
\end{tabular}

We note that all copula-based dependence measures are increasing functions of the copula parameters. We will work with the six copula families given in Table 1 which cover all types of tail dependence that can be found in the data.

\subsection{Copula Modeling of Serial Dependence}

A stylized fact about financial series is that current returns are usually affected only by their recent past. This makes the Markovianity property a plausible assumption for describing the evolution in time of moments of a returns series. As anticipated, 
the bivariate copula modeling of a random vector $\left(X_{1}, X_{2}\right)$ may now be adapted to model the conditional mean and volatility of a return series.

Let $r_{t}$ represent a stationary first order Markov process with continuous state space, and let $F_{1}$ denote its unconditional distribution function. Let $\left(r_{t}, r_{t+1}\right)$ be two consecutive random variables of this process. By Sklar's theorem, the process' properties are completely determined by the marginal distribution of $r_{t}$ and the copula function of $\left(r_{t}, r_{t+1}\right)$. This means that the transition distribution function is given by the conditional copula

$$
P\left(r_{t} \leq y_{t} \mid r_{t-1}=y_{t-1}\right)=C_{2 \mid 1}\left(F_{1}\left(y_{t}\right) \mid F_{1}\left(y_{t-1}\right)\right)
$$

where

$$
C_{2 \mid 1}(v \mid u)=P(V \leq v \mid U=u)=\frac{\partial C(u, v)}{\partial u},
$$

the first derivative of $C(\cdot)$.

Suppose now that a parametric copula family $C_{\psi}, \psi$ a parameter vector, and a marginal distribution $F_{1}$ indexed by a parameter vector $\alpha$ have been specified for the data at hand $r_{1}, r_{2}, \cdots, r_{T}$. We apply the full maximum likelihood method, and estimate marginal and association parameters simultaneously. The log-likelihood function to be jointly maximized is

$$
L\left(\alpha, \psi ; r_{1}, \ldots, r_{T}\right)=\sum_{t=1}^{T} L_{1, t}\left(\alpha ; r_{t}\right)+\sum_{t=2}^{T} L_{C, t}\left(\alpha, \psi ;\left(r_{t-1}, r_{t}\right)\right)
$$

where $L_{1, t}$ denotes the $\log$ likelihood function of $r_{t}$, and $L_{C, t}$ denotes the $\log$ of the copula density function. Maximization of (9) will lead to the joint maximum likelihood estimates (MLE) $(\widehat{\alpha}, \widehat{\psi})$.

After the estimation phase one usually wants to forecast quantities of interest using the conditional distribution of $r_{t}$ given its immediate past $r_{t-1}$. In finance, for example, the predictive distribution of future values may be used to compute one-step-ahead risk measures, or to estimate the entire asset price path over some fixed horizon.

To simulate a sequence of $T+1$ values from some Markov process and linked by a copula $C$, we iteratively apply the conditional copula (8): 
1. generate a random number $u_{1}$ from the uniform $(0,1)$ distribution such that, for any $v, 0 \leq C_{2 \mid 1}\left(v \mid u_{1}\right) \leq 1$;

2. iterate the following procedure $T+1$ times: generate $w_{k}$ from a uniform $(0,1)$ and compute $v_{k}$ as the numerical solution of the equation $C_{2 \mid 1}\left(v \mid u_{k}\right)=w_{k}$, where $u_{k}=v_{k-1}, k=1, \ldots, T+1$, with $u_{1}$ as defined in step 1 ;

3. transform the pairs $\left(u_{k}, v_{k}\right)$ back to their original values $\left(r_{t-1}, r_{t}\right)$ using the inverse function $F_{1}^{-1}$.

We note that the conditional copula approach may be applied to any time series. In the next section it is applied to the return series and to the squared return series, thus modeling the conditional mean and volatility of the assets.

\section{$3 \quad$ Fitting Conditional Copulas to U.S. stocks}

We provide applications based on the conditional copula modeling using daily returns on 80 stocks from the New York Stock Exchange. The period covered is February 28th, 2001, to February 17th, 2009, (length is 2002), obtained from the Bloomberg data base. The stocks are chosen from 8 different sectors: Basic Materials, Industrial Goods, Utilities, Technology, Services, Health Care, Financial, Consumer Goods, Conglomerates. For each sector we pick the 5 largest and the 5 smallest stocks in terms of market capitalization. However, some of these stocks were recent initial public offers and their series, being shorter than the others, were not used in the analysis. The final number of series analyzed is 62 .

As usual, we start the analysis by testing the returns series with respect to stationarity. The KPSS test rejected the null hypothesis for 3 series. They would require special treatment and were dropped from the analysis. Some basic statistics confirmed the well known stylized facts. All series are not normally distributed, possessing asymmetry and heavy tails, and according to the p-values of the LjungBox test (5\% level), 25 assets did not show significant autocorrelations in the first 3 lags. Although we have rejected the Ljung-Box null hypothesis for 34 series, as expected the strength of linear dependence between consecutive returns is very 
weak, as measured by the sample autocorrelation estimates. This is confirmed by the values of the empirical version of Spearman's rank correlation, which ranged from 0.01 to 0.14 .

The histograms of the squared returns revealed a non-strictly decreasing right asymmetric distribution. The squared returns series showed larger values for the rank correlation, ranging from 0.05 to 0.25 . The null hypothesis of no autocorrelation of the Ljung-Box test (in the second moment) was accepted for 6 assets.

\subsection{Conditional copula modelling of the mean and volatility}

As anticipated in the Introduction, we model the unconditional distribution of the log-returns with the skew- $t$ distribution (Hansen, 1994), an extention of the $t$-student distribution designed to capture asymmetry in the data. The skew- $t$ is a very flexible four parameter distribution which allows for calibrations in the location $(\mu)$, scale $(\sigma)$, tail thickness ( $v$, degrees of freedom) and skewness $(\lambda)$, while keeping the simplicity of the symmetric version. It has been successfully used when modeling financial returns, see Jondeau and Rockinger (2003) and Patton (2004, 2006).

Assuming the parametric families given in Table 1 (we also use the Clayton copula, which models lower tail dependence), we compute for each return series the joint maximum likelihood point estimates and standard errors of the parameters $(\alpha, \psi)$, where $\alpha=(\mu, \sigma, \lambda, v)$, and $\psi$ represents the copula parameters (see Table 1 ). The best fit was determined by the Akaike criterion. With respect to the marginal fits, we observed that the degrees of freedom are typically small (around 5 and 6) and that most skewness and location estimates are not statistically different from zero.

For all series the best copula fit turned out to be the $t$-copula. Since we are investigating Markov processes generated via tail dependent copulas, we tested (using the MLEs and their standard errors) and kept in the study the series possessing positive tail dependence between consecutive observations. Out off the 59 series, only 43 had the tail dependence coefficient statistically different from zero. The stocks names along with their sectors and classification as liquid or illiquid, and as small or large capitalizations are given in columns 1-3 of tables 2 and 3, where most 
relevant results are gathered.

In columns 4 and 5 we give the point estimates and standard errors of the $t$ copula parameter $\rho$ and corresponding tail dependence coefficient. It is interesting to note that, based on the $95 \%$ confidence interval constructed with the maximum likelihood estimates, most of these 43 series accepted the null hypothesis of zero lag 1 auto-correlation. The 5 exceptions showed $\rho$ negative and around -0.07 . They are the stocks SPF, CE Franklim, DNA, Pfiser, Gen Corp, and, as we will see later, for these stocks the copula approach for risk estimation performed better.

Next we conditionally model the second moment fitting copulas to the squared returns $r_{t}^{2}$. This strategy may be seen as an alternative to the ARCH(1) model of Engle (1982), which specifies that the conditional variance $\sigma_{t}^{2}$ at time $t$ follows an autoregressive model:

$$
\sigma_{t}^{2}=\alpha_{0}+\alpha_{1} r_{t-1}^{2}
$$

Specifying an $\mathrm{ARCH}(1)$ model for the return $r_{t}$ is equivalent to specifying $\mathrm{AR}(1)$ model for the squared returns. Applying copulas to consecutive squared returns, besides estimating the lag 1 autoregressive coefficient effect, also captures dependence in the tails. In other words, one is able to model clusters of extreme consecutive squared data even when this temporal dependence is not observed for small or moderate returns (usual days). The larger the tail dependence coefficient of the copula the greater the risk of persistence of high volatility in the series. One can expect to improve the volatility forecasts when using the copula approach since it uses information provided by the whole dependence structure between consecutive squared returns. Alternatives to the $\operatorname{ARCH}(r)$ generalization, $r \geq 1$, can be easily implemented by fitting $(r+1)$-dimensional copulas.

In order to fit copulas to the consecutive squared data we again combine the 6 parametric families given in Table 1 and take as unconditional marginal model the modified generalized Pareto distribution (MGPD) ${ }^{2}$. The initial candidate for modeling the unconditional distribution of the squared data was the Generalized Pareto distribution (GPD), but the shape of the squared data empirical distribution did not

\footnotetext{
${ }^{2} X$ follows a MGPD if $X^{b}, b>0$, follows a GPD. To deal with long tails usually $b<1$.
} 
match the required strictly decreasing behavior. Therefore a power transformation was necessary, what is equivalent to fit the MGPD.

As expected the best copula fits were provided by the Gumbel and the Survival Clayton copulas, those possessing upper tail dependence $\lambda_{U}$, meaning that high volatility days come in clusters. In columns 6 through 8 of tables 2 and 3 we provide part of the results concerning the modeling of the second moment.

For each stock we give the winning copula family (column labeled Copula(2)), and the point estimates and standard errors of the copula parameter and upper tail dependence coefficient. For all series the generalized log-likelihood test rejected the simple model (product copula) and all $\lambda_{U}$ are statistically different from zero. This means that a model for squared returns should explicitly account for dependence between consecutive high volatility days. However, our objective is to model the dynamics in the volatility, and an inherent problem when modeling and forecasting conditional volatility is that it is unobservable, and an issue is if the squared returns would be the best proxy for the variance. We intend in future work to compute the realized variance (Andersen, Bollerslev, Diebold, and Labys (2001, 2003)) using intra-day returns and apply the conditional copula modeling to this proxy.

Finally, to assess the effect of volatility in the dependence structure of consecutive returns we previously filter the returns series through Generalized Autoregressive Conditional Heteroscedasticity (GARCH) type models (Bollerslev, 1986), and then check for changes in conditional copula fits using the standardized residuals. The GARCH specifications included a leverage term, long memory, and were based on the normal and $t$-student conditional distributions. The best model was decided based upon the AIC values. The results obtained are not shown here but may be obtained from the authors by request. We observed that typically the strength of linear and non-linear serial dependence are weaker for the filtered data. For most stocks the copula family changed to the product copula. For the remaining series, although the copula parameters estimates were close to the independence boundary, the generalized likelihood test rejected the null hypothesis of independence. 


\section{Applications: Computing the Value at Risk}

We now investigate whether or not an important application in finance, namely the Value-at-Risk (VaR), can be improved by applying the proposed methodologies. The VaR measures the potential loss of a market position over a fixed horizon with a certain probability. It is the most popular tool for assessing market risk and it is basically a quantile of the asset probability distribution.

We compute the out-of-sample one-step-ahead VaR at the $1 \%$ and $5 \%$ risk levels for the most recent 250 observations in each series listed in tables 2 and 3 . When simulating the trajectories, we use the best conditional models (copulas and marginal distributions) found for each series, and obtain the one-step ahead predictive returns distribution, specified as the skew- $t$ distribution. Having the mean and variance of the skew- $t$ we compute the desired quantile, the VaR. The final one-step-ahead VaR value is the average value over 1000 simulations. For comparisons purpose, we also compute the Gaussian ARCH(1)-based VaR. The performances of the new copulabased VaR and of the traditional ARCH-based VaR are assessed through Kupiec's test at the $5 \%$ significance level (Kupiec, 1995).

Using the 250 VaR estimates we compute the Kupiec's test p-value and report in columns 9 and 10 of tables 2 and 3 a "C" or "A", when the null hypothesis of true risk $(1 \%$ and $5 \%)$ is accepted for the copula-based VaR or for the ARCH-based VaR, respectively. The notation -- is used when both methods fail on the test. Out of the 43 series, and for the $1 \%$ VaR, no procedure wins over the other. For the $5 \% \mathrm{VaR}$ and independently of the stocks' type (L/NL/La/Sm), the copula approach wins 14 times, the $\mathrm{ARCH}$ approach wins 10 times, both provide good results for 12 series, and both fail for 7 series. In summary, at the $1 \%$ risk level, the copula-based $\mathrm{VaR}$ is as good as the $\mathrm{ARCH}(1)-\mathrm{VaR}$, but at the $5 \%$ risk level the conditional copula approach provided better one-step-ahead VaR forecasts. 
Table 2: Summary of results from the conditional copula fits to the first and second moments, along with results from the out-of-sample VaR estimation.

\begin{tabular}{|c|c|c|c|c|c|c|c|c|c|}
\hline Stock & Sector & $\begin{array}{c}\mathrm{L} / \mathrm{NL} \\
\mathrm{La} / \mathrm{Sm}\end{array}$ & $\begin{array}{c}\rho \\
\operatorname{se}(\rho)\end{array}$ & $\begin{array}{c}\lambda_{L}=\lambda_{U} \\
\operatorname{se}\left(\lambda_{U}\right)\end{array}$ & Copula (2) & $\begin{array}{c}\theta \\
\operatorname{se}(\theta)\end{array}$ & $\begin{array}{c}\lambda_{U} \\
\operatorname{se}\left(\lambda_{U}\right)\end{array}$ & VaR $1 \%$ & VaR 5\% \\
\hline \multirow[t]{2}{*}{ CNOOC } & B. Materials & NL & -0.0191 & 0.0624 & S-Clayton & 0.2598 & 0.069 & - & - \\
\hline & & $\mathrm{La}$ & 0.0439 & 0.0167 & & 0.0010 & 0.000 & & \\
\hline \multirow[t]{2}{*}{ Petrobras } & B. Materials & $\mathrm{L}$ & -0.0172 & 0.0534 & S-Clayton & 0.2310 & 0.050 & - & - \\
\hline & & $\mathrm{La}$ & 0.0332 & 0.0166 & & 0.0008 & 0.000 & & \\
\hline \multirow[t]{2}{*}{ Lockheed M } & B. Materials & $\mathrm{L}$ & -0.0414 & 0.0644 & Gumbel & 1.1195 & 0.143 & - & - \\
\hline & & $\mathrm{Sm}$ & 0.0412 & 0.0148 & & 0.0003 & 0.000 & & \\
\hline \multirow[t]{2}{*}{ Emerson E } & Ind. Goods & $\mathrm{L}$ & -0.0014 & 0.0656 & S-Clayton & 0.2608 & 0.070 & $\mathrm{CA}$ & $\mathrm{C}$ \\
\hline & & $\mathrm{La}$ & 0.0412 & 0.0183 & & 0.0010 & 0.001 & & \\
\hline \multirow[t]{2}{*}{ Honeywell } & Ind. Goods & $\mathrm{L}$ & -0.0020 & 0.0488 & S-Clayton & 0.2472 & 0.061 & $\mathrm{CA}$ & $\mathrm{CA}$ \\
\hline & & $\mathrm{Sm}$ & 0.0380 & 0.0194 & & 0.0011 & 0.001 & & \\
\hline \multirow[t]{2}{*}{ SPF } & Ind. Goods & $\mathrm{L}$ & -0.0669 & 0.0528 & S-Clayton & 0.3412 & 0.131 & $\mathrm{CA}$ & $\mathrm{C}$ \\
\hline & & $\mathrm{Sm}$ & 0.0291 & 0.0123 & & 0.0013 & 0.001 & & \\
\hline \multirow[t]{2}{*}{ LMI } & Ind. Goods & $\mathrm{L}$ & -0.0048 & 0.0725 & S-Clayton & 0.2653 & 0.073 & - & $\mathbf{A}$ \\
\hline & & $\mathrm{Sm}$ & 0.0364 & 0.0140 & & 0.0012 & 0.001 & & \\
\hline \multirow[t]{2}{*}{ Hawk } & Ind. Goods & NL & -0.0227 & 0.0476 & S-Clayton & 0.2486 & 0.062 & $\mathrm{CA}$ & - \\
\hline & & $\mathrm{Sm}$ & 0.0425 & 0.0152 & & 0.0009 & 0.000 & & \\
\hline \multirow[t]{2}{*}{ Exelon } & Ind. Goods & $\mathrm{L}$ & -0.0262 & 0.0680 & S-Clayton & 0.2536 & 0.065 & $\mathrm{CA}$ & $\mathrm{CA}$ \\
\hline & & Ls & 0.0352 & 0.0107 & & 0.0010 & 0.001 & & \\
\hline \multirow[t]{2}{*}{ FPL } & Utilities & $\mathrm{L}$ & 0.0000 & 0.0693 & Gumbel & 1.1649 & 0.187 & $\mathbf{A}$ & $\mathbf{A}$ \\
\hline & & $\mathrm{La}$ & 0.0307 & 0.0151 & & 0.0004 & 0.000 & & \\
\hline \multirow[t]{2}{*}{ National G } & Utilities & $\mathrm{NL}$ & -0.0150 & 0.0504 & Gumbel & 1.1355 & 0.159 & - & $\mathbf{A}$ \\
\hline & & $\mathrm{La}$ & 0.0350 & 0.0190 & & 0.0003 & 0.000 & & \\
\hline \multirow[t]{2}{*}{ Semitool } & Technology & NL & -0.0016 & 0.0471 & Gumbel & 1.1201 & 0.143 & $\mathrm{CA}$ & $\mathbf{A}$ \\
\hline & & $\mathrm{Sm}$ & 0.0352 & 0.0143 & & 0.0003 & 0.000 & & \\
\hline \multirow[t]{2}{*}{ Zygo } & Technology & NL & -0.0369 & 0.0556 & Gumbel & 1.1445 & 0.168 & $\mathrm{CA}$ & $\mathrm{C}$ \\
\hline & & $\mathrm{Sm}$ & 0.0364 & 0.0158 & & 0.0003 & 0.000 & & \\
\hline \multirow[t]{2}{*}{ Technitrol } & Technology & NL & 0.0083 & 0.0508 & S-Clayton & 0.2971 & 0.097 & $\mathrm{CA}$ & $\mathrm{CA}$ \\
\hline & & $\mathrm{Sm}$ & 0.0329 & 0.0177 & & 0.0012 & 0.001 & & \\
\hline \multirow[t]{2}{*}{ Maxcom } & Technology & $\mathrm{NL}$ & -0.0435 & 0.0522 & Gumbel & 1.1416 & 0.165 & $\mathrm{CA}$ & $\mathrm{CA}$ \\
\hline & & $\mathrm{Sm}$ & 0.0415 & 0.0163 & & 0.0003 & 0.000 & & \\
\hline \multirow[t]{2}{*}{ CE Franklin } & Services & NL & -0.0900 & 0.0542 & Gumbel & 1.1172 & 0.140 & - & $\mathbf{A}$ \\
\hline & & $\mathrm{Sm}$ & 0.0346 & 0.0148 & & 0.0003 & 0.000 & & \\
\hline \multirow[t]{2}{*}{ Wal-Mart } & Services & $\mathrm{L}$ & -0.0414 & 0.0387 & S-Clayton & 0.3253 & 0.119 & $\mathrm{CA}$ & $\mathrm{CA}$ \\
\hline & & $\mathrm{La}$ & 0.0407 & 0.0159 & & 0.0013 & 0.001 & & \\
\hline \multirow[t]{2}{*}{ Guangshen } & Services & NL & -0.0392 & 0.0549 & Gumbel & 1.1379 & 0.161 & $\mathrm{CA}$ & $\mathrm{C}$ \\
\hline & & $\mathrm{Sm}$ & 0.0349 & 0.0171 & & 0.0003 & 0.000 & & \\
\hline \multirow[t]{2}{*}{ McDonald's } & Services & $\mathrm{L}$ & -0.0032 & 0.0544 & Gumbel & 1.1424 & 0.166 & $\mathrm{C}$ & $\mathbf{A}$ \\
\hline & & $\mathrm{La}$ & 0.0335 & 0.0180 & & 0.0003 & 0.000 & & \\
\hline \multirow[t]{2}{*}{ CVS } & Services & $\mathrm{L}$ & -0.0419 & 0.0407 & Gumbel & 1.1605 & 0.183 & $\mathrm{CA}$ & $\mathrm{CA}$ \\
\hline & & $\mathrm{La}$ & 0.0273 & 0.0149 & & 0.0003 & 0.000 & & \\
\hline \multirow[t]{2}{*}{ Johnson\&J } & Healthcare & $\mathrm{L}$ & -0.0502 & 0.0463 & Gumbel & 1.1420 & 0.165 & $\mathrm{CA}$ & $\mathrm{C}$ \\
\hline & & $\mathrm{La}$ & 0.0415 & 0.0187 & & 0.0003 & 0.000 & & \\
\hline
\end{tabular}


Table 3: Continued: Summary of results from the conditional copula fits.

\begin{tabular}{|c|c|c|c|c|c|c|c|c|c|}
\hline \multirow[t]{2}{*}{ DNA } & \multirow[t]{2}{*}{ Healthcare } & NL & -0.0688 & 0.0391 & \multirow[t]{2}{*}{ Gumbel } & 1.1252 & 0.148 & \multirow[t]{2}{*}{$\mathrm{CA}$} & \multirow[t]{2}{*}{$\mathrm{C}$} \\
\hline & & $\mathrm{La}$ & 0.0391 & 0.0178 & & 0.0003 & 0.000 & & \\
\hline \multirow[t]{2}{*}{ Pfizer } & \multirow[t]{2}{*}{ Healthcare } & $\mathrm{L}$ & -0.0921 & 0.0437 & \multirow[t]{2}{*}{ Gumbel } & 1.1336 & 0.157 & \multirow[t]{2}{*}{$\mathrm{CA}$} & \multirow[t]{2}{*}{$\mathrm{C}$} \\
\hline & & $\mathrm{La}$ & 0.0343 & 0.0193 & & 0.0003 & 0.000 & & \\
\hline \multirow[t]{2}{*}{ GlaxoSmith } & \multirow[t]{2}{*}{ Healthcare } & $\mathrm{L}$ & 0.0199 & 0.0598 & \multirow[t]{2}{*}{ Gumbel } & 1.1425 & 0.166 & \multirow[t]{2}{*}{ CA } & \multirow[t]{2}{*}{$\mathrm{C}$} \\
\hline & & $\mathrm{Sm}$ & 0.0397 & 0.0180 & & 0.0003 & 0.000 & & \\
\hline \multirow[t]{2}{*}{ Mannatech } & \multirow[t]{2}{*}{ Healthcare } & NL & -0.0347 & 0.0586 & \multirow[t]{2}{*}{ Gumbel } & 1.1196 & 0.143 & \multirow[t]{2}{*}{$\mathrm{CA}$} & \multirow[t]{2}{*}{$\mathrm{C}$} \\
\hline & & $\mathrm{Sm}$ & 0.0370 & 0.0190 & & 0.0003 & 0.000 & & \\
\hline \multirow[t]{2}{*}{ WEA } & \multirow[t]{2}{*}{ Financial } & NL & -0.0039 & 0.0568 & S-Clayton & 0.3764 & 0.159 & $\mathrm{CA}$ & $\mathrm{CA}$ \\
\hline & & $\mathrm{Sm}$ & 0.0385 & 0.0162 & & 0.0013 & 0.001 & & \\
\hline Boulder & Financial & NL & -0.0188 & 0.0577 & S-Clayton & 0.2625 & 0.071 & $\mathrm{CA}$ & $\overline{\mathrm{CA}}$ \\
\hline & & $\mathrm{Sm}$ & 0.0418 & 0.0190 & & 0.0011 & 0.001 & & \\
\hline 1st Financ & Financial & NL & -0.0121 & 0.0545 & Gumbel & 1.1683 & 0.190 & $\mathrm{CA}$ & $\mathbf{A}$ \\
\hline & & $\mathrm{La}$ & 0.0369 & 0.0183 & & 0.0003 & 0.000 & & \\
\hline Unibanco & Financial & $\mathrm{L}$ & -0.0373 & 0.0479 & Gumbel & 1.1545 & 0.177 & - & - \\
\hline & & $\mathrm{La}$ & 0.0443 & 0.0147 & & 0.0003 & 0.000 & & \\
\hline JPMorgan & Financial & $\mathrm{L}$ & -0.0045 & 0.0462 & Gumbel & 1.1701 & 0.192 & $\mathrm{CA}$ & $\mathbf{A}$ \\
\hline & & $\mathrm{La}$ & 0.0361 & 0.0140 & & 0.0003 & 0.000 & & \\
\hline WellsFargo & Financial & $\mathrm{L}$ & -0.0401 & 0.0467 & Gumbel & 1.1353 & 0.159 & $\mathrm{CA}$ & $\mathbf{A}$ \\
\hline & & $\mathrm{La}$ & 0.0393 & 0.0171 & & 0.0003 & 0.000 & & \\
\hline$P \& G$ & Consumer Goods & $\mathrm{L}$ & 0.0046 & 0.0761 & Gumbel & 1.1764 & 0.197 & - & - \\
\hline & & $\mathrm{La}$ & 0.0411 & 0.0174 & & 0.0003 & 0.000 & & \\
\hline Toyota & Consumer Goods & $\mathrm{L}$ & -0.0359 & 0.0606 & S-Clayton & 0.2458 & 0.060 & $\mathrm{CA}$ & - \\
\hline & & $\mathrm{La}$ & 0.0362 & 0.0170 & & 0.0009 & 0.000 & & \\
\hline Honda & Consumer Goods & $\mathrm{L}$ & 0.0349 & 0.0686 & Gumbel & 1.1237 & 0.147 & $\mathrm{CA}$ & $\mathrm{C}$ \\
\hline & & $\mathrm{La}$ & 0.0362 & 0.0166 & & 0.0003 & 0.000 & & \\
\hline Oxfords & Consumer Goods & NL & -0.0175 & 0.0443 & S-Clayton & 0.2848 & 0.088 & $\mathrm{CA}$ & $\mathrm{C}$ \\
\hline & & $\mathrm{Sm}$ & 0.0290 & 0.0141 & & 0.0010 & 0.001 & & \\
\hline A Axle & Consumer Goods & $\mathrm{L}$ & -0.0172 & 0.0542 & Gumbel & 1.1495 & 0.172 & $\mathbf{A}$ & $\mathbf{A}$ \\
\hline & & $\mathrm{Sm}$ & 0.0325 & 0.0123 & & 0.0003 & 0.000 & & \\
\hline Crane & Conglomerates & NL & -0.0325 & 0.0419 & Gumbel & 1.1504 & 0.173 & $\mathrm{CA}$ & $\mathrm{C}$ \\
\hline & & $\mathrm{La}$ & 0.0428 & 0.0187 & & 0.0004 & 0.000 & & \\
\hline GenCorp & Conglomerates & NL & -0.0684 & 0.0473 & S-Clayton & 0.3226 & 0.117 & $\mathrm{CA}$ & $\mathrm{CA}$ \\
\hline & & $\mathrm{Sm}$ & 0.0290 & 0.0141 & & 0.0014 & 0.001 & & \\
\hline Cherokee & Conglomerates & NL & -0.0443 & 0.0658 & S-Clayton & 0.2952 & 0.096 & $\mathrm{C}$ & $\mathrm{CA}$ \\
\hline & & $\mathrm{La}$ & 0.0325 & 0.0123 & & 0.0013 & 0.001 & & \\
\hline Rentech & Conglomerates & NL & -0.0186 & 0.0457 & S-Clayton & 0.2450 & 0.059 & $\mathrm{CA}$ & $\mathrm{CA}$ \\
\hline & & $\mathrm{Sm}$ & 0.0428 & 0.0187 & & 0.0010 & 0.000 & & \\
\hline GE & Conglomerates & NL & 0.0038 & 0.0608 & Gumbel & 1.1250 & 0.148 & $\mathrm{CA}$ & $\mathrm{C}$ \\
\hline & & $\mathrm{La}$ & 0.0396 & 0.0166 & & 0.0003 & 0.000 & & \\
\hline United Tech & Conglomerates & $\mathrm{L}$ & -0.0321 & 0.0587 & S-Clayton & 0.2639 & 0.072 & $\mathrm{CA}$ & $\mathrm{C}$ \\
\hline & & $\mathrm{La}$ & 0.0394 & 0.0162 & & 0.0012 & 0.001 & & \\
\hline Danaher & Conglomerates & $\mathrm{L}$ & -0.0195 & 0.0605 & S-Clayton & 0.2288 & 0.048 & $\mathrm{CA}$ & $\mathrm{CA}$ \\
\hline & & $\mathrm{Sm}$ & 0.0420 & 0.0125 & & 0.0009 & 0.000 & & \\
\hline
\end{tabular}




\section{Conclusions}

This paper used a conditional copula approach for modeling the time dependence in the mean and variance of a univariate time series. Motivation came from empirical evidence that financial returns present short memory which affects both the first and second moments in a linear and also non-linear fashion. Thus, the whole dependence structure linking consecutive returns and their squares was modeled using copulas, an strategy which allows to go beyond the time series linear models and/or the linear correlation coefficient.

To explore the conditional copula approach potentials we used a data set composed by 62 American stocks, finally analyzing just 43 possessing positive tail dependence. Inference was based on two-steps maximum likelihood. Several marginal distributions and copula families were considered for fitting. The $t$-copula was the best model for the returns, and the Gumbel or Survival Clayton provided best fits for the squared returns. For the marginal fits the best models turned out to be the skew- $t$ and the modified GPD distributions, respectively for the returns and their squares.

As expected, we found stronger association in the returns second moment when compared to the the first moment, as well as significant non-linear dependence, as measured by the upper tail dependence, explaining the volatility clusters.

Having captured and modeled the dependence between consecutive returns, one is able to simulate the returns' one-step-ahead predictive distribution, and applications follow. For example, one may be interested in obtaining the returns' future trajectory, or in estimating one-step-ahead risk measures. We computed the onestep-ahead VaR at the 1\% and 5\% risk levels for the 43 U.S. stocks, and compared to those obtained from an $\mathrm{ARCH}(1)$ fit. The $\mathrm{ARCH}(1)$ is the fair comparison since based on behavior of squared consecutive observations.

The Kupiec's test p-value was used to compare the out-of-sample copula-based and ARCH-based VaR, estimated for the last 250 observations. Out of the 43 series, and for the $1 \% \mathrm{VaR}$, no procedure wins over the other. However, for the 5\% VaR, the copula approach wins 14 times against the 10 times won by the $\mathrm{ARCH}$ approach. 
The other cases were ties. In summary, at the $1 \%$ risk level, the copula-based VaR is as good as the $\mathrm{ARCH}(1)-\mathrm{VaR}$, but at the $5 \%$ risk level the conditional copula approach provided better one-step-ahead VaR forecasts. This may be considered an indication that the modeling through copulas possessing positive tail dependence would provide better forecasts whenever there is persistence in the series provided by non-linear dependence between consecutive returns and their squares.

We focused on first order Markov processes. However, more information may be extracted from the data by considering higher dimensional copulas. They would be competitors for the $\mathrm{ARCH}(r), r>1$, models. We also note that simple extentions of the conditional copula model allows for including indicator functions as leverage terms to model the effect of consecutive negative (or positive) returns. Further, more flexibility can be added if conditional pair-copulas are considered. We intend to pursue this investigation in future work.

It is very interesting to have alternative methods for computing the VaR since it is well recognized today that the best methodology for VaR computing is data dependent. Our work indicates a new methodology to be further explored. Finally, time series analysis in many other areas such as epidemiology, environment, economy, and so on, may benefit from the conditional copula model approach.

\section{References}

Andersen, T. G.; Bollerslev, T.; Diebold, F. X. Labys, P. (2001) The distribution of realized exchange rate volatility, Journal of the American Statistical Association, 96(453):42-55.

Andersen, T. G.; Bollerslev, T.; Diebold, F. X. Labys, P. (2003) Modeling and forecasting realized volatility, Econometrica, 71(2):579-625.

Bollerslev, T. (1986). A Conditionally Heteroskedastic Time Series Model for Speculative Prices and Rates of Return, The Review of Economics and Statistics, Vol. 69, No. 3 (Aug., 1987), pp. 542-547.

Econometrics, 130, 307-335. 
Chen, Xiaohong \& Fan, Yanqin (2006). "Estimation and Model Selection of Semiparametric Copula-Based Multivariate Dynamic Models Under Copula Misspecification". Journal of Econometrics, 135(1-2), 125-154.

Chen, Xiaohong \& Fan, Yanqin \& Tsyrennikov, Viktor, 2006. "Efficient Estimation of Semiparametric Multivariate Copula Models". Journal of the American Statistical Association, vol. 101, pages 1228-1240.

Cherubini, U., Luciano, E., Vecchiato, W. (2004). Copula Methods in Finance, John Wiley and Sons, Chichester.

Domma, F., Giordano, S., Perri, P. F. (2008). Statistical Modelling of Temporal Dependence in Financial Data via Copula Function, Departement of Economic ans Statistics, University of Calabria.

Genest, C., and J. MacKay (1986a). The joy of copulas: Bivariate distributions with uniform marginals, The American Statistician, 40, 280-283.

Genest, C., and R. MacKay (1986b). Copules archimediennes et familles de lois bidimensionell dont les marges sont donnees, The Canadian Journal of Statistics, 14, 145-159.

Genest, C. and Rivest, L.P. (1993). Statistical inference procedures for bivariate Archimedian copulas, Journal of Amer. Statist. Assoc., 88, 423, 1034-1043.

Genest, C., M. Gendron and M. Bourdeau-Brien (2008). "The Advent of Copulas in Finance", forthcoming in European Journal of Finance.

Hansen, B. E. (1994). Autoregressive conditional density estimation, International Economic Review, Vol.35, No.3.

Ibragimov, R. (2009). "Copula based characterizations for higher-order markov processes". Available at http://www.economics.harvard.edu/ faculty/ibragimov/files/ IbragimovCopulasFinal.pdf.

Joe, H. (1997). Multivariate models and dependence concepts, Chapman \& Hall,London.

Jondeau, E., and M. Rockinger (2003). "Conditional volatility, skewness, and kurtosis: existence,persistence, and comovements". Journal of Economic Dynamics and Control, 27, 16991737. 
Kupiec, P. H. (1995). "Techniques for verifying the accuracy of risk measurement models". Journal of Derivatives, 2, 173184.

Nelsen, Roger B. (2006)., An Introduction to Copulas, second edition, Springer Series in Statistics.

Patton, A. (2004). "On the Out-of-Sample Importance of Skewness and Asymmetric Dependence for Asset Allocation". Journal of Financial Econometrics, 2(1), 130 168.

Patton, A. (2006). "Modelling Asymmetric Exchange Rate Dependence". International Economic Review, 47(2).

Schweizer, B.and A. Sklar (1983). Probabilistic Metric Spaces. North-Holland, N.York.

Sklar, A. (1996). Random variables, distribution functions, and copulas (a personal look backward and forward), in Distributions with Fixed Marginals and Related Topics, ed. by L. Rüschendor, B. Schweizer, and M. Taylor,. 1-14. IMS, Hayward, CA. 
